\title{
ORIGIN OF THE NEAR-ECLIPTIC CIRCUMSOLAR DUST BAND
}

\author{
David Nesvorný, ${ }^{1}$ William F. Bottke, ${ }^{1}$ David Vokrouhlický, ${ }^{2}$ Mark Sykes, ${ }^{3}$ David J. Lien, ${ }^{3}$ and John Stansberry ${ }^{4}$ \\ Received 2008 March 19; accepted 2008 April 8; published 2008 May 8
}

\begin{abstract}
The zodiacal dust bands are bright infrared (IR) strips produced by thermal emission from circumsolar rings of particles. Two of the three principal dust bands, known as $\beta$ and $\gamma$, were previously linked to the recent asteroid collisions that produced groups of fragments, so-called asteroid families, near the orbits of (832) Karin and (490) Veritas. The origin of the third, near-ecliptic $\alpha$ band has been unknown until now. Here we report the discovery of a recent breakup of a $>20 \mathrm{~km}$ diameter asteroid near $\alpha$ 's originally suspected source location in the Themis family. Numerical modeling and observations of the $\alpha$-band thermal emission from the Spitzer Space Telescope indicate that the discovered breakup is the source of $\alpha$-band particles. The recent formation of all principal dust bands implies a significant time variability of the circumstellar debris disks.
\end{abstract}

Subject headings: interplanetary medium — minor planets, asteroids

\section{INTRODUCTION}

The zodiacal cloud (ZC) is a thick circumsolar disk of dust particles produced by asteroid collisions and comets that contribute to it in unknown proportions. The thermal IR emission of the ZC is brightest near the ecliptic, where the $25 \mu \mathrm{m}$ fluxes are 30-100 $\mathrm{MJy} \mathrm{sr}^{-1}$, and extends all the way to the ecliptic poles (Hausen et al. 1984). Important clues to the origin of ZC are provided by structures known as zodiacal dust bands that were discovered using observations from the Infrared Astronomical Satellite (IRAS; Low et al. 1984). The $\alpha, \beta$, and $\gamma$ bands form small-scale latitude features in the zodiacal light. They are thought to be produced by thermal emission of three pairs of circumsolar dust rings near inclinations $i=1.4^{\circ}$, $2.1^{\circ}$, and $9.3^{\circ}$, respectively (Sykes 1988, 1990). These dust rings were originally proposed to be sustained by ongoing collisional grinding of fragments in the prominent Themis, Koronis, and Eos asteroid families (Dermott et al. 1984) because these large families, remnants of ancient catastrophic asteroid collisions, have roughly the right orbital inclinations.

More recently, it was found that the $\gamma$ band does not arise from the Eos family (Grogan et al. 2001), but instead traces the slightly smaller orbital inclination of the Veritas family (Dermott et al. 2002; Nesvorný et al. 2003), which is a remnant of the catastrophic breakup of a diameter $D>150 \mathrm{~km}$ asteroid $\approx 8.2$ Myr ago (Nesvorný et al. 2003; Farley et al. 2006). Moreover, the Karin family, which formed by breakup of a $D \approx$ $40 \mathrm{~km}$ Koronis family member $\approx 5.8 \mathrm{Myr}$ ago, was shown to produce the $\beta$ band (Nesvorný et al. 2002, 2006a). These findings raise the possibility that the near-ecliptic $\alpha$ dust band may also arise from a recent asteroid disruption.

As first noted by Sykes (1990), the observed latitude of the $\alpha$ band precisely corresponds to the mean inclination of the Themis family, which formed by a disruption of a $D \sim 350 \mathrm{~km}$ asteroid >1 Gyr ago (Marzari et al. 1995; Nesvorný et al. 2003; Durda et al. 2007). Dust production within a family is expected to decline many orders of magnitude over billions of years

\footnotetext{
${ }^{1}$ Department of Space Studies, Southwest Research Institute, 1050 Walnut Street, Suite 300, Boulder, CO 80302.

${ }^{2}$ Institute of Astronomy, Charles University, V Holešovičkách 2, CZ-18000, Prague 8, Czech Republic.

${ }^{3}$ Planetary Science Institute, 1700 East Fort Lowell, Suite 106, Tucson, AZ 85719.

${ }^{4}$ California Institute of Technology, Spitzer Science Center, 1200 East California Boulevard, Pasadena, CA 91125.
}

(e.g., Sykes \& Greenberg 1986; Bottke et al. 2005). Therefore, it is puzzling that IRAS would detect the infrared surface brightness of a Themis dust band and not detect dust bands from dozens of other known large/old families.

\section{OBSERVATIONS}

To clarify this issue, we used the Spitzer Space Telescope to observe the diffuse thermal emission of the $\mathrm{ZC}$ near the ecliptic. Four sets (A, B, C, D) of four parallel scans (1, 2, 3, 4) were obtained with the $24 \mu \mathrm{m}$ MIPS array. The scans were $5.4^{\prime}$ wide and went from $+10^{\circ}$ down to $-10^{\circ}$ in the ecliptic latitude, $b$. Individual scans in each set are roughly parallel to each other across the ecliptic and are separated by $1.5^{\circ}$ in ecliptic longitude, $l$. By co-adding all 128 pixels cross-scan, the MIPS images were used to generate "noodles" with a 6.4 " in-scan resolution that show profiles of the diffuse infrared flux in the $24 \mu \mathrm{m}$ wavelength as a function of $b$. These profiles provide $\approx 10$ times better sensitivity and $\approx 20$ times better spatial resolution than IRAS observations. Additional information about our observations, including the time and pointing direction, can be found in Nesvorný et al. (2006b).

We used a Fourier filter to enhance small-scale structures in the noodles (Reach et al. 1997). The filter suppressed features of the signal with latitudinal spreads $<0.1^{\circ}$ and $>4^{\circ}$. It removed the broad and bright background emission associated with the $\mathrm{ZC}$ background. The best results were obtained for scan set D where parallax from the high solar elongation of observations resulted in the clear separation of the $\alpha$ and $\beta$ bands in $b$ (Fig. 1).

We numerically modeled the collisional and orbital evolution of asteroid particles and used our Synthetic InfraRed Telescope code (SIRT; see Nesvorný et al. 2006a) to test whether the location and width of the observed $\alpha$-band peaks can be matched by the thermal emission of dust from various asteroidal sources. We found that none of the known suspected sources with $i \sim 1.4^{\circ}$, including the Themis and Massalia families (Nesvorný et al. 2003), provides a satisfactory match (Fig. 1).

\section{THE BEAGLE FAMILY}

By analyzing the newest AstDyS catalog of asteroid orbits ${ }^{5}$ (Milani \& Knežević 1994) we have serendipitously discovered a new asteroid family with mean $i=1.34^{\circ}$. The new family

\footnotetext{
${ }^{5}$ See http://hamilton.dm.unipi.it/cgi-bin/astdys/astibo.
} 


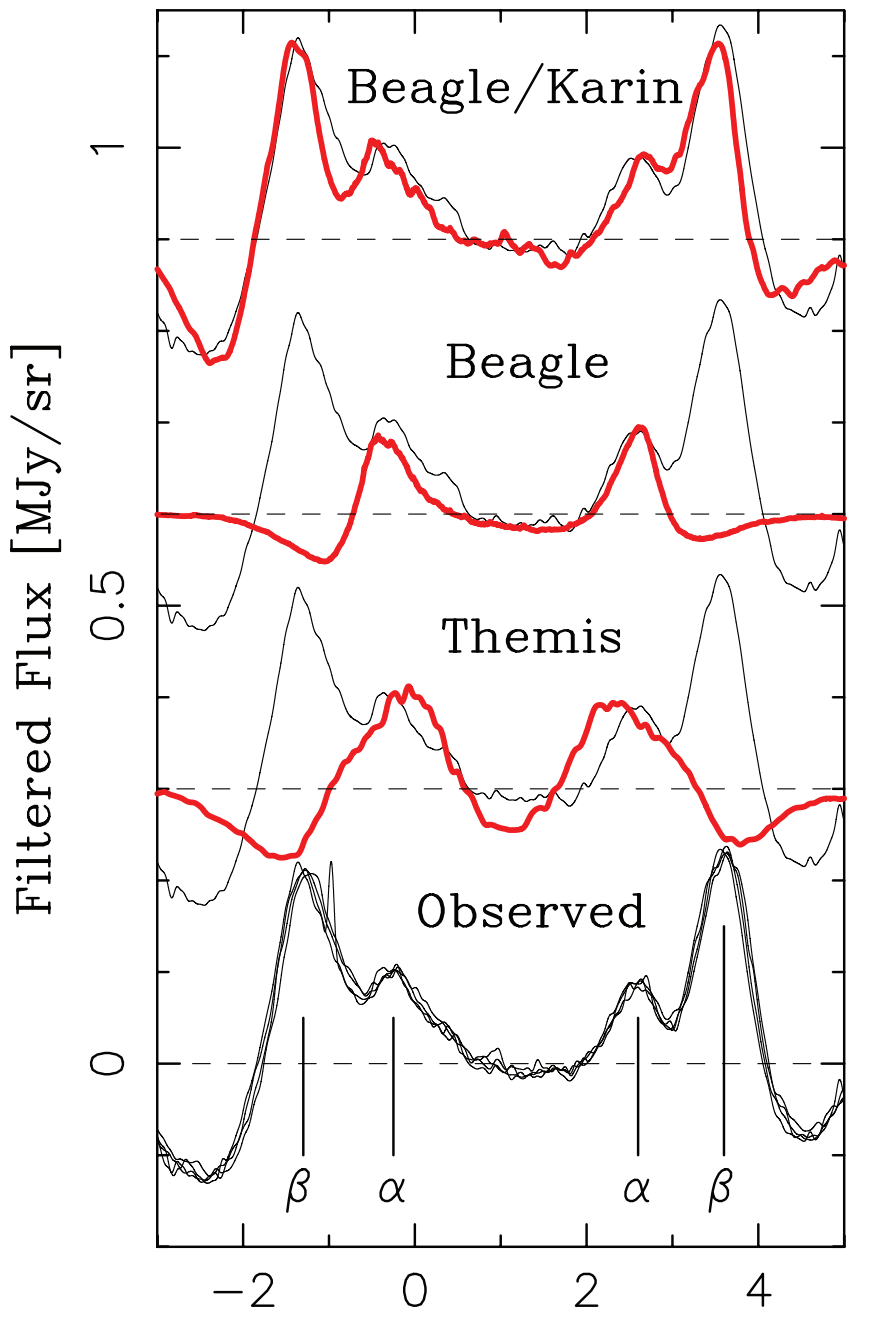

Ecliptic Latitude (deg)

FIG. 1.-Comparison of our Spitzer observations of the $\alpha$ dust band and model profiles for various sources shows that the Beagle family is the source of the $\alpha$ dust band. Bottom lines: The $\alpha$ and $\beta$ dust bands appear in our four filtered D-set scans as pairs of emission peaks near latitudes $b=-0.3^{\circ}$ and $2.6^{\circ}$ and $b=-1.3^{\circ}$ and $3.6^{\circ}$, respectively. Above we show comparisons of the D1 scan to profiles determined with SIRT for various sources: Themis, Beagle, and combined Beagle/Karin model (red lines). These profiles are offset by $0.3 \mathrm{MJy} \mathrm{sr}^{-1}$ for clarity. The model peaks for Themis particles are broader and closer to the ecliptic than the observed ones. Conversely, the model profile for Beagle particles nicely matches the $\alpha$ band emission. A two-source model with Karin and Beagle particles (top) provides an excellent fit to the observed near-ecliptic features.

is located at $a=3.157$ AU near the orbit of $D=53 \mathrm{~km}$ Themis-family asteroid (656) Beagle (Fig. 2). This large asteroid may not be a real member of the family due to a slight eccentricity offset of its orbit from other identified fragments (unusual ejection velocity field would have to be invoked otherwise). Despite this problem, we will refer to the new family as the Beagle family, a name pending revision when the exact family membership becomes more secure.

Interestingly, (7968) Elst-Pizzaro (Elst et al. 1996), whose comet-like activity was observed in 1996 and 2002 (Elst et al. 1996; Hsieh \& Jewitt 2006), joins the Beagle family if relaxed but still plausible membership criteria are adopted (Fig. 2). In total, we identified $\approx 65$ dynamical members of the Beagle family whose diameters range down to $D \approx 2 \mathrm{~km}$ (corresponding

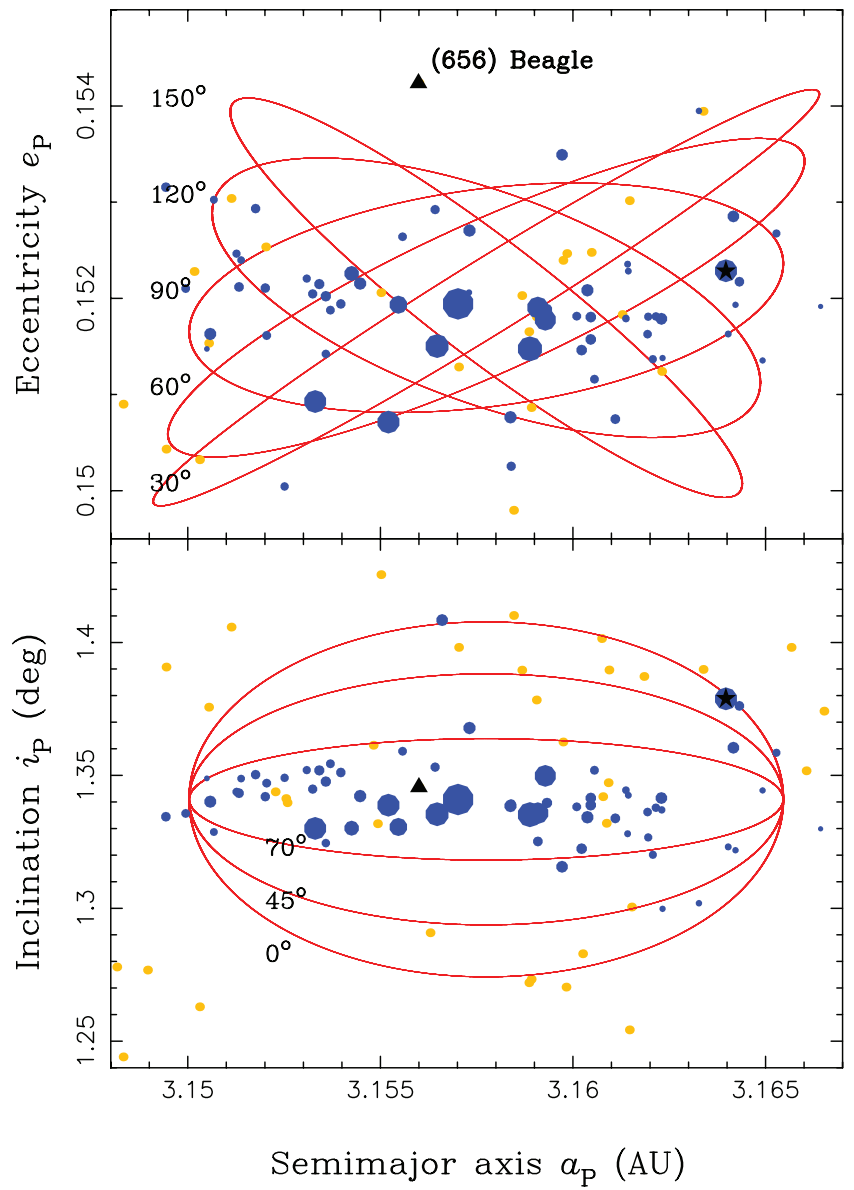

FIG. 2.-Proper orbital elements, $a_{P}, e_{P}$, and $i_{P}$, of Beagle family asteroids are tightly clustered, indicating that the family-forming event occurred recently. Top: Projection of orbits into $\left(a_{P}, e_{P}\right)$-plane. Bottom: Projection into $\left(a_{P}, i_{P}\right)$-plane. We show 65 identified members of the Beagle family (blue symbols) and background Themis family asteroids (yellow symbols). The size of a blue symbol is proportional to asteroid size. The orbits of (656) Beagle and (7968) Elst-Pizzaro are indicated by a triangle and star, respectively. Red ellipses show expected location of fragments launched from $a_{P}=3.1574 \mathrm{AU}$, $e_{P}=0.1520$, and $i_{P}=1.341^{\circ}$ with $20 \mathrm{~m} \mathrm{~s}^{-1}$ speeds. Numbers in the top and bottom panels denote assumed values of $f$ and $f+\omega$, respectively, where $f$ is the true anomaly and $\omega$ is the perihelion argument.

to their absolute magnitudes $H=17$ and Themis-family mean albedo $A=0.054$; Tedesco et al. 2002). The total volume obtained by combining estimated volumes of all identified fragments indicates a $D>20 \mathrm{~km}$ disrupted parent object [or $D>$ $65 \mathrm{~km}$ if (656) Beagle is a member].

The Beagle family has a tight spread in semimajor axis, $\Delta a=0.013 \mathrm{AU}$. This suggests that it must have formed very recently. Old families are typically much larger in $a$ because their orbits become dispersed in $a$ over time by a radiation recoil force known as the Yarkovsky effect (Bottke et al. 2006). To place an upper limit on the Beagle family's age, we assumed that all ejected fragments started with $a=3.157 \mathrm{AU}$ and drifted over time $t$ with constant speed $d a / d t=10^{-4} \cos \epsilon / D$ AU $\mathrm{Myr}^{-1}$ with $D$ in $\mathrm{km}$. Here, the constant was computed for $D=1 \mathrm{~km}$ using the linear theory of Yarkovsky drag (Vokrouhlický 1999) and $\epsilon$ is the obliquity.

As the fragments drift in $a$, the full width of our test family increases as $\Delta a(D)=2 \times 10^{-4} t / D$ AU with $t$ in Myr. (Here we neglect changes of daldt of individual fragments due to impacts and radiation torques. These changes should be min- 
imal on the considered timescale [e.g., Vokrouhlický et al. 2006].) We determined $\Delta a(D)$ by fitting an envelope to the observed distribution of the Beagle family in $(a, D)$ and found that $t \approx 50 \mathrm{Myr}$ is required to explain it. Therefore, the Beagle family's age, $t_{\text {age }}$, must be $<50 \mathrm{Myr}$, because a major part of its current semimajor axis spread must have been contributed by the ejection speeds in the immediate aftermath of the breakup.

To determine $t_{\text {age }}$ more precisely, we numerically integrated the Beagle family orbits backward in time to show their convergence at $t_{\text {age }}$. Similar methods were used to date the Karin and Veritas families (Nesvorný et al. 2002, 2003). Here, however, the method is complicated by the proximity of the Beagle family to the $2: 1$ resonance with Jupiter which produces large gradients of secular frequencies with $a$ (e.g., $d g / d a \approx 0.3^{\circ}$ $\mathrm{yr}^{-1} \mathrm{AU}^{-1}$ where $g$ is the perihelion longitude frequency). Tiny changes in $a$, such as the ones produced by the Yarkovsky effect, can affect the secular frequencies in ways that are difficult to track unless additional information becomes available about $\epsilon$ values and other parameters that determine the $d a / d t$ values of individual Beagle family asteroids.

To overcome this difficulty, we tracked the past evolution of 21 clone orbits for each of 26 numbered Beagle asteroids. The orbits started within the orbital uncertainty region around the nominal orbit and sampled all theoretically possible values of da/dt. In total, we followed 546 orbits over 50 Myr. The integration included the gravitational effects of all planets except Mercury. We found several solutions for $t_{\text {age }}<10 \mathrm{Myr}$ (Fig. 3; e.g., $t_{\text {age }} \approx 6.3 \mathrm{Myr}$ ). We thus believe that the Beagle family is probably $\$ 10$ Myr old or only slightly older. This age is similar to those of the Karin (5.8 Myr; Nesvorný et al. 2002) and Veritas families (8.3 Myr; Nesvorný et al. 2003).

\section{MODEL}

We used the SIRT code to determine the expected thermal signal for populations of dust particles produced in the Beagle family. Radiating particles were assumed to be isothermal spheres with a range of diameters, $D$ (see Nesvorný et al. 2006a). Material constants of low-temperature cosmic pyroxene (Henning \& Mutschke 1997) were used to determine the emissivity and equilibrium temperature of dust particles as a function of heliocentric distance, $h$. Their orbital and size distributions were calculated from $N$-body integrations and a Monte Carlo model for the collisional cascade (Nesvorný et al. 2006a). The collisional lifetimes of particles were set as a function of $D$ and $h$ based on results of interplanetary dust impact experiments on spacecraft detectors (Grün et al. 1985).

The model signal from the Beagle family shows an excellent fit to our Spitzer observations of the $\alpha$ dust band (Fig. 1). The key factor in obtaining this result was the tight inclination spread of the Beagle family, $\Delta i \approx 0.05^{\circ}$. In contrast, the Themis family with $\Delta i \approx 1.2^{\circ}$ would produce the IR emission distribution that is much broader in latitude than the observed one. This shows that the recently formed Beagle family, and not the old Themis family, is the real source of the $\alpha$ dust band.

Using SIRT, we were able to constrain the total number, cross section, and volume of Beagle dust particles. The total cross section is $\approx(0.4-0.6) \times 10^{9} \mathrm{~km}^{2}$, which represents a value about 5-10 smaller than that of Karin particles in the $\beta$ dust band. This difference probably stems from the larger size of the Karin parent body and/or a slightly longer $t_{\text {age }}$ of the Beagle family. Combined, the cross sections of Beagle and Karin particles amount for $\approx(2.3-3) \times 10^{9} \mathrm{~km}^{2}$, which is a $\approx 2$ times

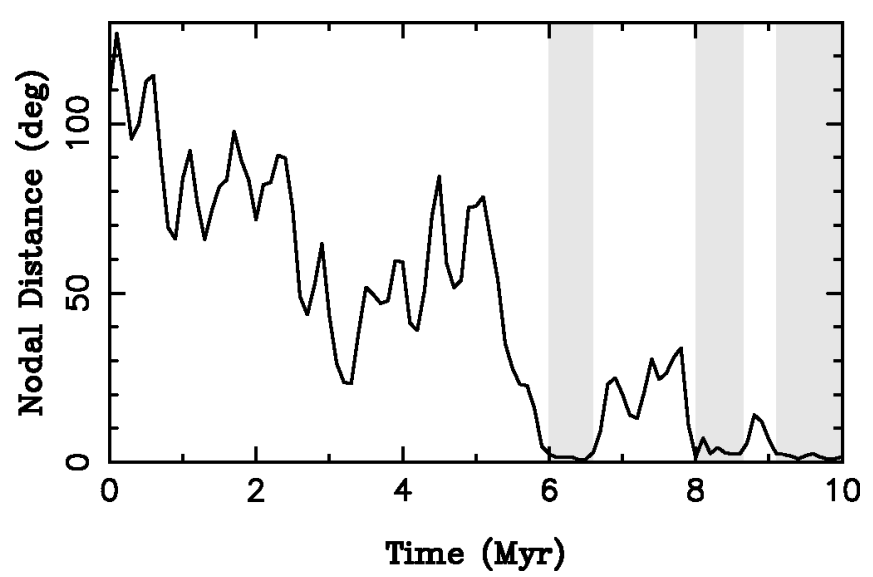

FIG. 3.-Past convergence of nodal longitudes indicates that the Beagle family probably formed by a recent breakup that occurred $\lesssim 10 \mathrm{Myr}$ ago. We show here the mean nodal distance between pairs of orbits of individual Beagle family asteroids in the past $10 \mathrm{Myr}$. The values at different time epochs $t$ were obtained by direct $N$-body integration of orbits backward in time. Convergences of nodal longitudes occur for $t \approx 6.3, \approx 8.3$, and $\approx 9.1 \mathrm{Myr}$ ago (denoted by gray regions). Unfortunately, an age for the Beagle family exceeding $10 \mathrm{Myr}$ cannot be strictly excluded by our method due to the uncertain Yarkovsky drift rates of individual bodies. It is also difficult to demonstrate the convergence of perihelion longitudes because of their large gradient with $a$ and uncertain drift rates. Note that age $<50 \mathrm{Myr}$ is indicated by the tight semimajor axis spread of the family.

lower value than the one determined previously (Nesvorný et al. 2006a). (We believe that the larger cross section obtained for $\alpha$ and $\beta$ dust bands by Nesvorný et al. was probably an artifact of their method because they attempted to fit the unresolved IRAS observations of $\alpha$ and $\beta$ by Karin particles only.) The new estimated cumulative volumes of Beagle and Karin particles with $1 \mu \mathrm{m}<D<1 \mathrm{~cm}$ correspond to $D \approx 6$ and 9 $\mathrm{km}$ spheres, respectively. Due to smaller $i$ and larger gravitational focusing of Beagle particles by Earth, the Earth-accretion rates of Beagle and Karin particles should be roughly comparable, amounting for estimated total of $\sim 30 \%$ of the measured total accretion rate of interplanetary dust particles by the Earth (Love \& Brownlee 1993).

\section{CONCLUSIONS}

These results have important implications for the understanding of the contribution of asteroid sources to the circumsolar dust cloud and the origin of circumstellar debris disk in general. The absence of dust bands detected for old asteroid families implies that a population of asteroid dust particles like those in the observed dust bands cannot be sustained by continuous collisional grinding over $\gg 10$ Myr. Instead, the observed dust bands were produced by large stochastic breakups within the past $~ 10$ Myr. We estimate that the overall contribution of these recent breakups to the $\mathrm{ZC}$ is small, probably $\lesssim 10 \%$. Other sources must be responsible for the remaining $\gtrsim 90 \%$.

Our work may also explain the current activity of (7968) Elst-Pizzaro (Elst et al. 1996; Hsieh \& Jewitt 2006). If this object is indeed a fragment of the identified breakup, we hypothesize that the volatile-rich carbonaceous material from the interior of the disrupted asteroid may have been initially exposed on the Elst-Pizzaro's surface. Over time, this material would have become shielded from the solar radiation by an increasingly thicker volatile-poor surface layer. After 10 Myr, however, the layer may not be still thick enough to protect the subsurface ices from occasional small impacts or thermally driven bursts. We speculate that other active asteroids observed 
in the outer main belt (Hsieh \& Jewitt 2006) may also be fragments of recent breakups of volatile-rich asteroids.

This Letter is based on work supported by the NASA's Planetary Geology and Geophysics program and Spitzer Cycle 1 grant entitled "The Production of Zodiacal Dust by Asteroids and Comets" (PID 2317, PI Sykes). The work of D. V. was supported by research grant 205/08/0064 of the Czech Grant Agency. We thank William Reach, Daniel Durda, Sumita Jayaraman, and Russell Walker for their contributions to planning and obtaining the Spitzer observations.

\section{REFERENCES}

Bottke, W. F., Durda, D., Nesvorný, D., Jedicke, R., Morbidelli, A., Vokrouhlický, D., \& Levison, H. 2005, in IAU Colloq. 197, Dynamics of Populations of Planetary Systems, ed. Z. Knezevic \& A. Milani (Cambridge: Cambridge Univ. Press), 357

Bottke, W. F., Jr., Vokrouhlický, D., Rubincam, D. P., \& Nesvorný, D. 2006, Annu. Rev. Earth Planet. Sci., 34, 157

Dermott, S. F., Kehoe, T. J. J., Durda, D. D., Grogan, K., \& Nesvorný, D. 2002, in Proc. Asteroids, Comets, Meteors, ed. B. Warmbein (ESA SP-500; Noordwijk: ESA), 319

Dermott, S. F., Nicholson, P. D., Burns, J. A., \& Houck, J. R. 1984, Nature, 312,505

Durda, D. D., Bottke, W. F., Nesvorný, D., Enke, B. L., Merline, W. J., Asphaug, E., \& Richardson, D. C. 2007, Icarus, 186, 498

Elst, E. W., Pizarro, O., Pollas, C., Tichá, J., Tichý, M., Moravec, Z., Offutt, W., \& Marsden, B. G. 1996, IAU Circ., 6456, 1

Farley, K. A., Vokrouhlický, D., Bottke, W. F., \& Nesvorný, D. 2006, Nature, 439, 295

Grogan, K., Dermott, S. F., \& Durda, D. D. 2001, Icarus, 152, 251

Grün, E., Zook, H. A., Fechtig, H., \& Giese, R. H. 1985, Icarus, 62, 244

Hausen, M. G., et al. 1984, ApJ, 278, L15
Henning, T., \& Mutschke, H. 1997, A\&A, 327, 743

Hsieh, H. H., \& Jewitt, D. 2006, Science, 312, 561

Love, S. G., \& Brownlee, D. E. 1993, Science, 262, 550

Low, F. J., et al. 1984, ApJ, 278, L19

Marzari, F., Davis, D., \& Vanzani, V. 1995, Icarus, 113, 168

Milani, A., \& Knežević, Z. 1994, Icarus, 107, 219

Nesvorný, D., Bottke, W. F., Levison, H., \& Dones, L. 2002, Nature, 417, 720

- 2003, ApJ, 591, 486

Nesvorný, D., Vokrouhlický, D., Bottke, W. F., \& Sykes, M. 2006a, Icarus, 181,107

Nesvorný, D., et al. 2006b, AJ, 132, 582

Reach, W. T., Franz, B. A., \& Weiland, J. L. 1997, Icarus, 127, 461

Sykes, M. V. 1988, ApJ, 334, L55 1990, Icarus, 85, 267

Sykes, M. V., \& Greenberg, R. 1986, Icarus, 65, 51

Tedesco, E. F., Egan, M. P., \& Price, S. D. 2002, AJ, 124, 583

Vokrouhlický, D. 1999, A\&A, 344, 362

Vokrouhlický, D., Brož, M., Bottke, W. F., Nesvorný, D., \& Morbidelli, A. 2006, Icarus, 182, 118 